\title{
Three Dimensional Near Infrared Tomography of the Breast
}

\author{
Matthew E Eames ${ }^{1}$, Brian W Pogue ${ }^{2}$, Colin M Carpenter ${ }^{2}$ and Hamid Dehghani ${ }^{1}$ \\ ${ }^{1}$ School of Physics, University of Exeter, UK \\ 2 Thayer School of Engineering, Dartmouth College, Hanover, NH 03755, USA
}

Near-Infrared (NIR) Diffuse Optical Tomography (DOT) is a non-invasive imaging technique which is used to obtain functional and physiological images of soft tissue, such as the female breast, specifically for the detection and characterization of breast cancer. The vast majority of the work to date has been limited to two dimensional (2D) models which have provided valuable insight into tissue function and physiology enabling a better understanding of tumor development and treatment. Although the 2D image reconstruction approach is fast and computationally efficient, it has limitations as it does not correctly represent the volume under investigation and therefore do not provide the most accurate model for image reconstruction. Three dimensional (3D) modeling and image reconstruction is becoming more accessible through the development of sophisticated numerical models and computationally fast algorithms. A robust and general method is presented which reconstructs 3D functional images using a more accurate and realistic spectral model of 3D light propagation in tissue. Results from a single patient example are presented to demonstrate the clinical importance of 3D image reconstruction in optical tomography for the detection and characterization of breast cancer.

\section{Introduction}

Near-Infrared (NIR) optical tomography is a non-invasive imaging technique which aims at recovering the functional and optical parameters of tissue being imaged [1]. NIR light of wavelength between $650 \mathrm{~nm}$ and $900 \mathrm{~nm}$ is injected into the tissue being imaged using optical fibers at the surface of the imaging volume and the emergent light is measured at points on the same surface using either other fibers or a non-contact imaging detector array. This boundary data is then used together with a model based image reconstruction algorithm, to either derive the intrinsic optical properties of the tissue at the applied wavelengths [2], or to derive functional information such as total hemoglobin content and water fraction using spectral measurements [3].

The transport of light in tissue at these wavelengths becomes nearly isotropic in scatter direction after a few scattering distances and is well predicted by a diffuse model of the photons [2, 4]. Application of diffuse optical tomography has become an important research tool and is potentially emerging as a non-invasive imaging technique for quantifying brain functional activity in children [5] and adults [6], as well as breast cancer detection and characterization [1, 7, 8].

This paper will outline the three dimensional (3D) Finite Element Model (FEM) and image reconstruction algorithm, NIRFAST, which can derive functional information about the volume under investigation using measured NIR boundary data. The theory behind the model based problem formulation is described as well as the image reconstruction algorithms used. Results from a single patient example are presented to demonstrate the clinical importance of 3D image reconstruction on optical tomography.

\section{Theory}

\subsection{The forward model}

When the magnitude of the isotropic fluence within tissue is significantly larger than the directional flux magnitude, the light field is "diffuse". This occurs when the scattering interaction dominates over absorption in a region of interest far from sources and boundaries, and when the light fluence is not rapidly changing with time (i.e. such as in the subpicosecond time regime). This assumption allows a transition from the Boltzmann transport equation, which is used to describe an anisotropic light field, to the diffusion equation approximation [2]. The diffusion approximation in the frequency domain is given by 
$-\nabla \cdot \kappa(\mathbf{r}) \nabla \Phi(\mathbf{r}, \omega)+\mu_{a}(\mathbf{r})+\frac{i \omega}{c_{m}(\mathbf{r})} \Phi(\mathbf{r}, \omega)=q_{0}(\mathbf{r}, \omega)$

where $q_{0}(\mathbf{r}, \omega)$ is an isotropic source, $\Phi(\mathbf{r}, \omega)$ is the photon fluence rate at position $\mathbf{r}$ and modulation frequency $\omega . \kappa$ is the diffusion coefficient given by

$\kappa=1 / 3\left(\mu_{a}+\mu_{s}^{\prime}\right)$,

where $\mu_{a}$ and $\mu_{s}^{\prime}$ are absorption and reduced scattering (or transport scattering) coefficients respectively. $c_{m}(\mathbf{r})$ is the speed of light in the medium at any point, defined by $c_{\mathrm{o}} / n(\mathbf{r})$, where $n(\mathbf{r})$ is the index of refraction at the same point and $c_{0}$ is the speed of light in vacuum.

The air-tissue boundary is derived with an index-mismatched type III condition, in which the fluence at the edge of the tissue exits and does not return. The flux leaving the external boundary is equal to the fluence rate at the boundary weighted by a factor that accounts for the internal reflection of light back into the tissue. This relationship is described by

$\Phi(\xi, \omega)+2 A \hat{\mathbf{n}} \cdot \kappa(\xi) \nabla \Phi(\xi, \omega)=0$

where $\xi$ is a point on the external boundary, and $A$ depends upon the relative refractive index (RI) mismatch between the tissue domain and air which can be derived from Fresnel's law

$A=\frac{2 /\left(1-R_{0}\right)-1+\left|\cos \theta_{c}\right|^{3}}{1-\left|\cos \theta_{c}\right|^{2}}$,

where $\theta_{c}=\arcsin \left(n_{A I R} / n_{1}\right)$, the angle at which total internal reflection occurs for photons moving from the tissue region with RI $n_{1}$ to air with RI $n_{\text {AIR }}$, and $R_{0}=\left(n_{1} / n_{\text {AIR }}-1\right)^{2} /\left(n_{1} / n_{\text {AIR }}+1\right)^{2}$. At the external boundaries, generally the RI is that of free space, so that $n_{\text {AIR }}=1$.

\subsection{Single wavelength image reconstruction}

The inverse problem is solved with the aim of recovering the optical parameters, $\mu=\left(\mu_{a}, \kappa\right)$, for each node within the volume of interest. The inversion is achieved by minimizing the difference between the measured data, $\Phi^{M}$, and the calculated data, $\Phi^{C}$, using a modified-Tikhonov minimization approach given by

$$
\Omega=\sum_{\mu}^{\min }\left\{\sum_{i=1}^{n m}\left(\Phi_{i}^{M}-\Phi_{i}^{C}\right)+\lambda \sum_{j=1}^{n n}\left(\mu_{j}-\mu_{0}\right)^{2}\right\},
$$

where $n m$ is the number of measurements and $n n$ is the number of FEM nodes or unknowns and $\lambda$ is the Tikhonov regularization parameter. $\mu_{\mathrm{o}}$ is either an initial guess of the optical properties, generally obtained by data-calibration procedure [9], or an a-priori optical property distribution. It has been found that providing the initial guess $\mu_{\mathrm{o}}$ is not too far from the actual parameter distribution, this term can be ignored [10]. 
Minimizing equation (5) with respect to the optical parameters, involves setting the first order differential equation $\frac{\delta \Omega}{\delta \mu}$ equal to zero. Taking the linear approximation and solving iteratively using the Levenberg-Marquardt procedure, assuming $\delta \mu=\mu_{i}-\mu_{0}$, we get

$\left(J^{T} J+\lambda I\right)^{-1} J^{T} \delta \Phi=\delta \mu$

$\delta \mu$ is the update for the optical properties and $\delta \Phi$ is the current iteration data-model misfit. $I$ is the identity matrix. The Jacobian, $J$, sometimes referred to as the sensitivity matrix, defines the relationship between small changes in boundary data and small changes in optical properties. The format of the Jacobian has been published previously and has dimensions number of measurements (phase and $\log$ amplitude) by 2 times the number of unknowns [8]. $\lambda$ is implemented in a method where it starts at a given value ( $\lambda=1000$ in the presented study) scaled by maximum of diagonal of $J^{T} J$ and is systematically reduced at each iteration. The iterative procedure is repeated until experimental data matches the modeled data within a tolerance level, which is typically set at $2 \%$.

From these single wavelength reconstructions, chromophore concentrations are calculated using a constrained least squares fit to the Beer's law relation. An empirical approximation to Mie scattering theory is used to estimate model parameters scatter amplitude (a) and scatter power (b) with wavelength in $\mu \mathrm{m}$.

\subsection{Spectral image reconstruction}

Alternatively, it is possible incorporate the physiological parameters into the reconstruction and directly reconstruct for oxyhemoglobin, de-oxyhemoglobin, water, scatter amplitude and scatter power, thus reducing the parameter space from two times the number of wavelengths ( $\mu_{a}$ and $\kappa$ at each wavelength) to 5 parameters [3]. This is achieved with a simple extension to equation (6) where the Jacobian now relates a change in boundary data to a change in chromophore concentration and scattering parameters. The new Jacobian has the form

$$
J_{c, \lambda}=\left.\frac{\partial \Phi}{\partial c}\right|_{\lambda}=\left.\frac{\partial \Phi}{\partial \mu_{a}} \cdot \frac{\partial \mu_{a}}{\partial c}\right|_{\lambda}
$$

The Jacobian now has the dimensions of number of wavelengths times number of measurements per wavelength by number of nodes times number of wavelength-independent parameters. However, since the size of the Jacobian is now dominated by a much larger number of unknowns, the Moore-Penrose generalized inverse is found to be better suited, which is the case for an under-determined problem. This gives rise to the update equation

$J^{T}\left(J J^{T}+\lambda I\right)^{-1} J^{T} \delta \Phi=\delta \mu$

where $\delta \mu$ is now the update vector consisting of the chromophore concentration and the scattering properties. This approach is easily extendable to additional wavelengths and chromophores. The value of $\lambda$ is equal to 10 for the studies presented..

\section{Results}

In this section we present a case report of a 29 year old woman with a $2 \mathrm{~cm}$ infiltrating ductal carcinoma located mediocaudally in the left breast. In this study, simultaneous Magnetic Resonance Imaging (MRI) and NIR spectroscopy data were collected. The NIR boundary data were acquired from a coronal section over the location of the tumor, using 16 sources and 16 detectors at a modulation frequency of 100Mhz. The instrument details are described elsewhere [10]. 
MRI of pre and post contrast injections were taken and shown in figure 1. Figure 1 (a) shows the anatomical axial view of both entire breasts with contrast enhancement indicating the location of the tumor. Figure 1(b) shows the coronal view in the plane of the tumor before the injection and figure 1(c) shows the subtraction image of post-pre injection. From these images the abnormality within the left breast is clearly visible. The NIR spectroscopy measurements were taken at 6 wavelengths of 649, 761, 785, 808, 829 and $849 \mathrm{~nm}$ from 16 source/detector locations across an entire coronal slice of the breast giving rise to 480 data points per wavelength [11].

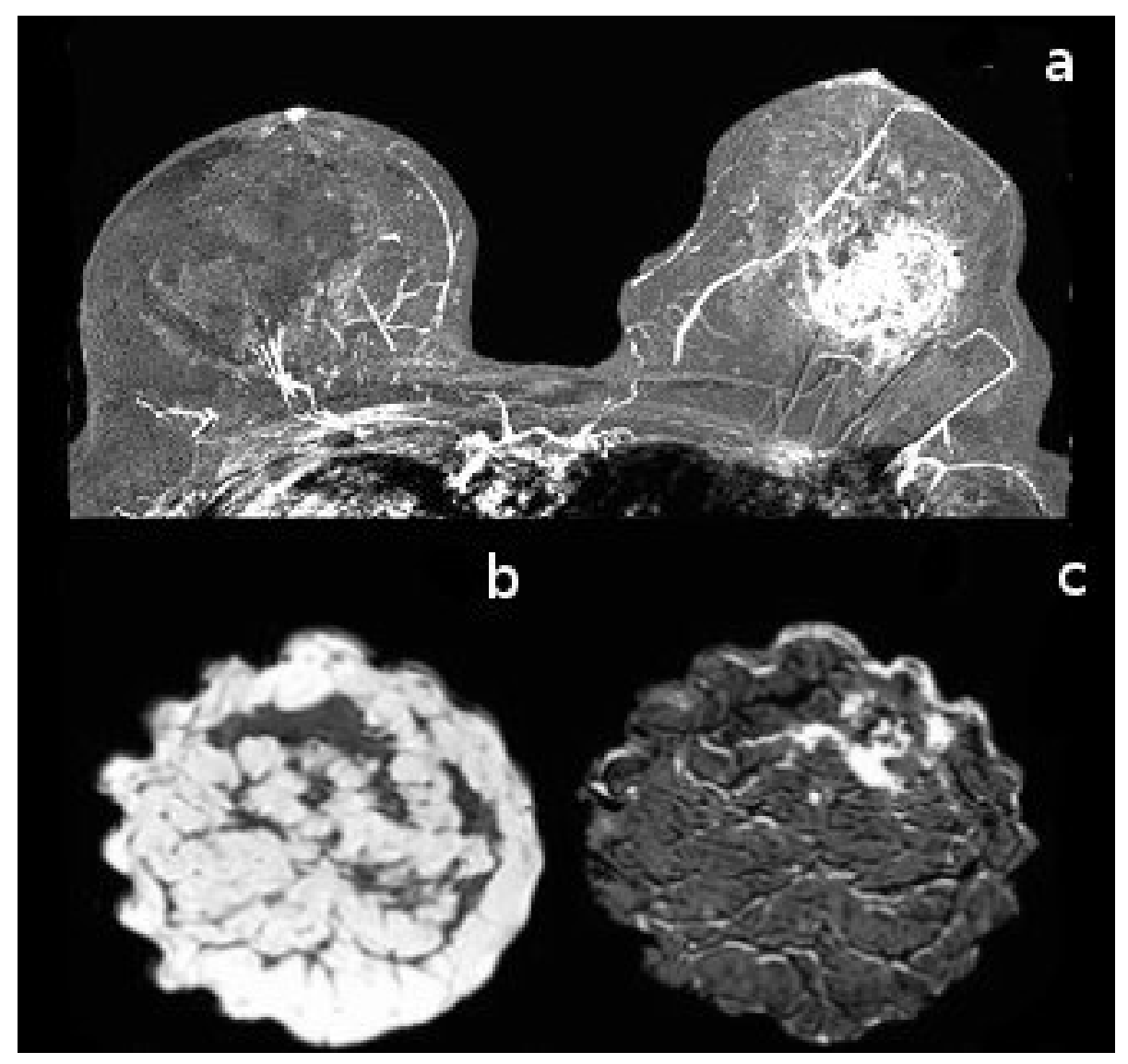

Figure 1. Magnetic Resonance images of the (a) anatomically axial images of both breasts with dynamic contrast resolution to show the location of the tumor. (b) Coronal view pre contrast enhancement in the plane of the tumor and (c) subtraction image of post-pre contrast enhancement injection.

For image reconstruction a 3D FEM mesh of the left breast is used as shown by figure 2 . This mesh was created by manually segmenting $2 \mathrm{D}$ coronal MRI slices to generate 3D surface maps. These maps in turn were used to create a volume mesh of the whole breast. The mesh consists of 37,198 nodes corresponding to 184,904 elements. Since good initial guess reconstruction parameters are crucial for image reconstruction, a data-model misfit algorithm is used [8]. Firstly global $\mu_{a}$ and $\mu_{s}^{\prime}$ values for the corresponding measured data at each wavelength are calculated based on the source-detector separation by use of an analytic formula for a homogeneous semi-infinite medium. This initial guess is used to calculate new global values with the FEM model of the volume. The average difference between the calculated fitted data and the measured data is then subtracted from the measured data which is used to calibrate the data for image 
reconstruction. All reconstructions were processed on a $3.7 \mathrm{GHz}$ workstation running Linux Redhat with 8 GBytes of RAM.

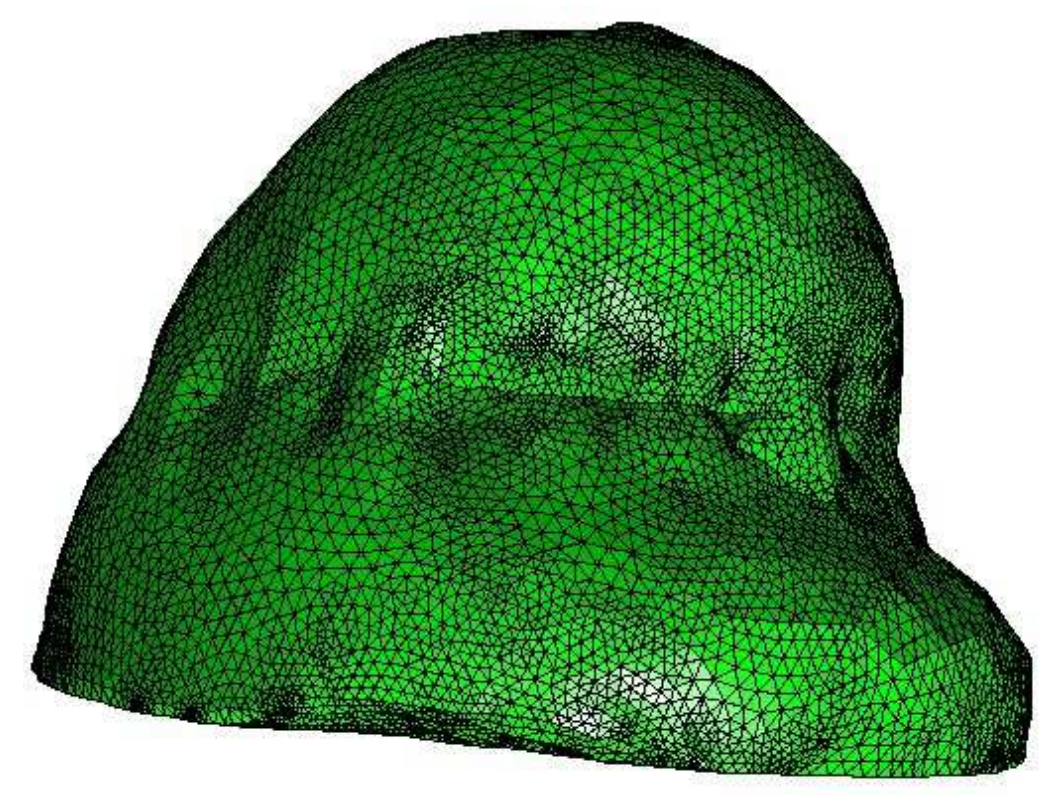

Figure 2. Three dimensional finite element mesh used for the image reconstructions.

\subsection{Single wavelength case}

In this study 6 different wavelengths of $661 \mathrm{~nm}, 761 \mathrm{~nm}, 785 \mathrm{~nm}, 808 \mathrm{~nm}, 826 \mathrm{~nm}$ and $849 \mathrm{~nm}$ were used. For each wavelength global values $\mu_{\mathrm{a}}$ and $\mu_{\mathrm{s}}^{\prime}$ were calculated as described above. Images were then reconstructed iteratively until the modeled data did not improve more than $2 \%$ as compared to the previous iteration. Each wavelength took between 1 and 10 iterations to complete and took 640s per iteration. Using Beer's law and an approximation to Mie Theory the scattering and absorption parameters are used to calculate the chromophore concentrations and scattering properties of the region of interest.

Figure 3 shows the reconstructed images for both coronal and axial views corresponding to the location of the reconstructed anomaly. These results show clearly the region of interest which correspond well to the MRI image of figure 1 . These reconstructions show a peak in the total hemoglobin and oxygen saturation, however a large negative peak in the water content is seen. This is because the chromophore concentrations are calculated by an unbounded least squares fit and therefore are not constrained to realistic positive values. Likewise the Scattering parameter reconstructions do not provide much information due to the high level of noise and unconstrained fitting of parameters. 


\section{Chromophore}

$\mathrm{H}_{\mathrm{b}} \mathrm{T}(\mathrm{mM})$

$\mathrm{SO}_{2}(\%)$

$\mathrm{H}_{2} \mathrm{O}(\%)$

\section{Scattering} amplitude

\section{Scattering power}
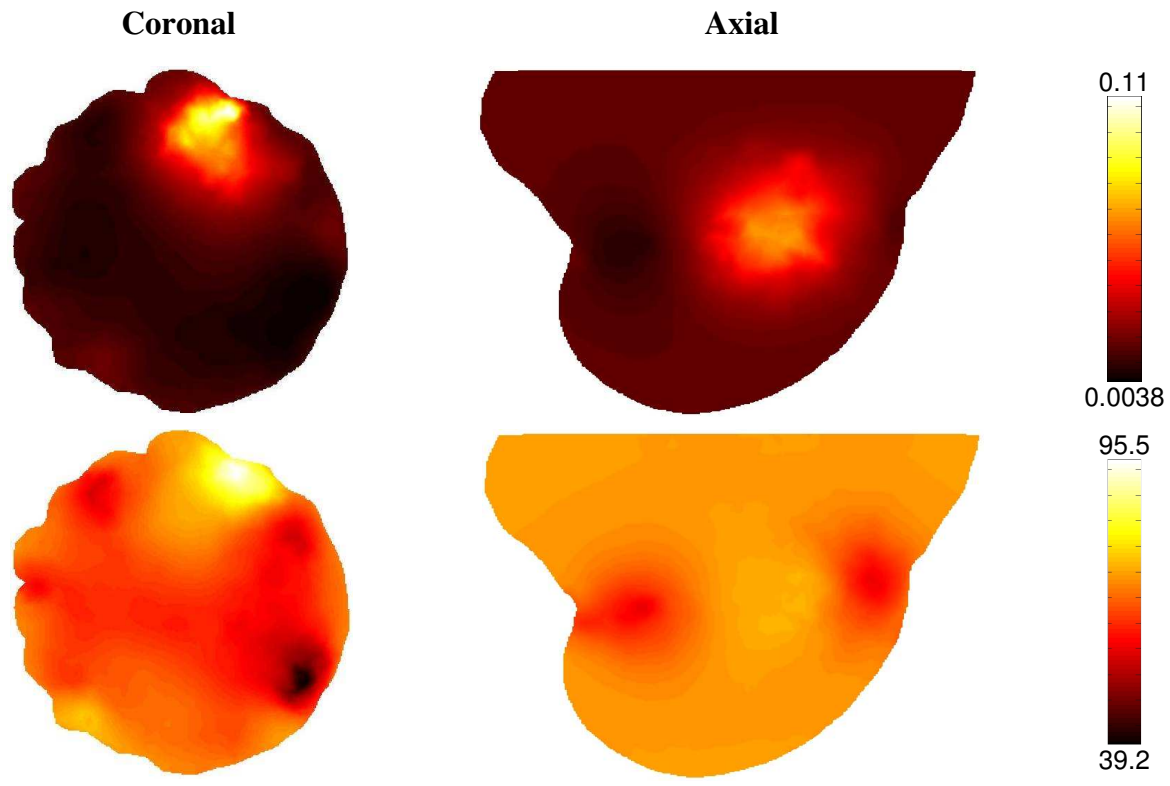

95.5

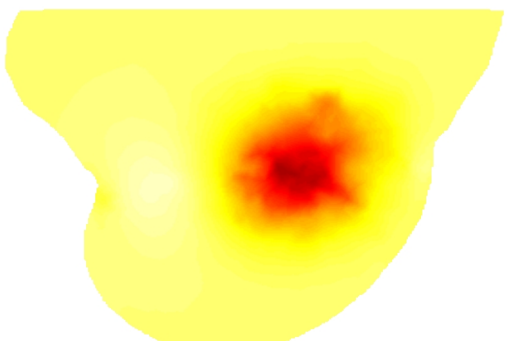

39.2

82.2
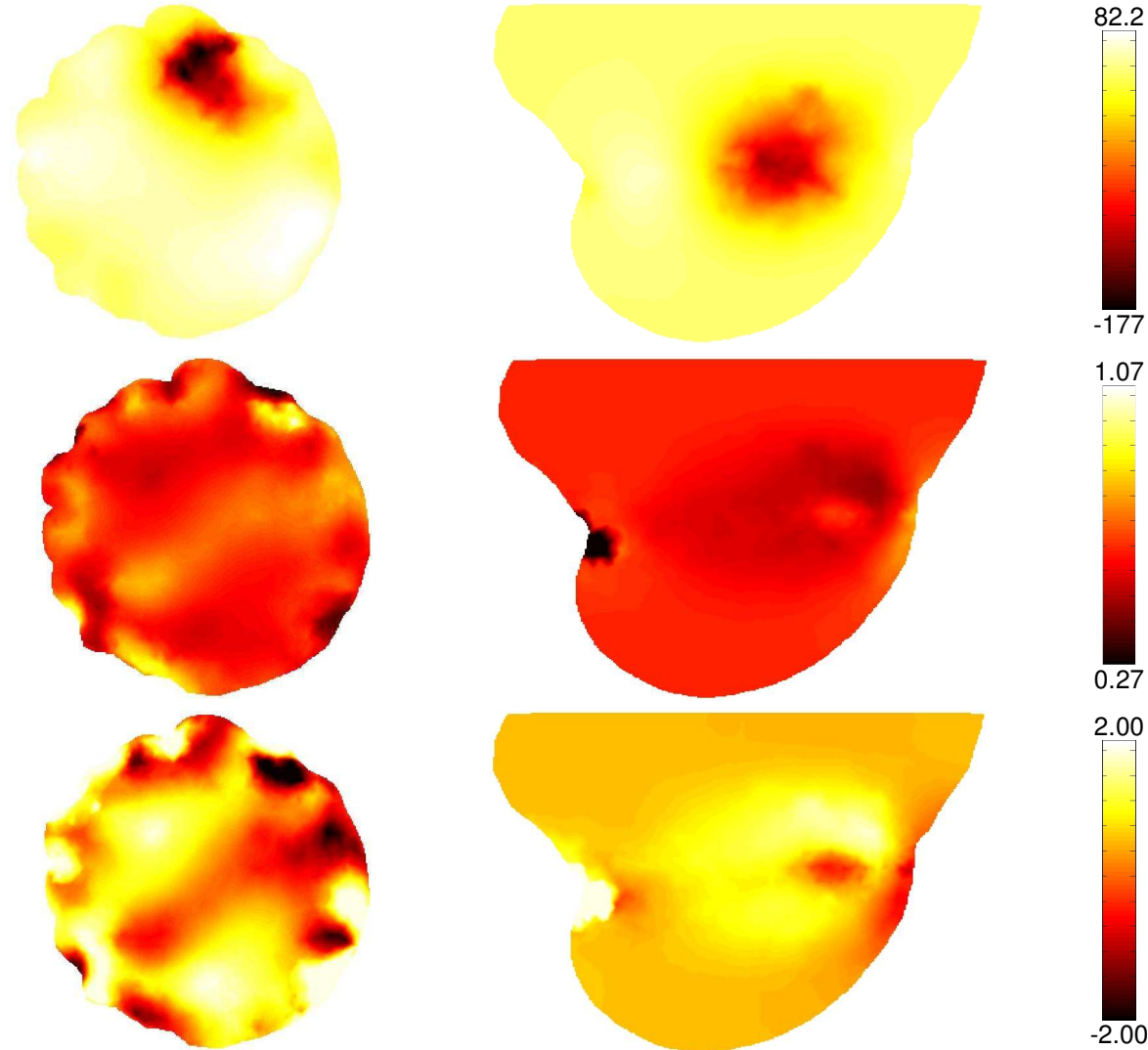

Figure 3. Reconstructed chromophore concentrations of total hemoglobin $\left(\mathrm{H}_{\mathrm{b}} \mathrm{T}\right)$, oxygen saturation $\left(\mathrm{SO}_{2}\right)$, water content $\left(\mathrm{H}_{2} \mathrm{O}\right)$, scattering amplitude and scattering power using 6 single wavelength reconstructions combined displayed as coronal and anatomically axial views. 


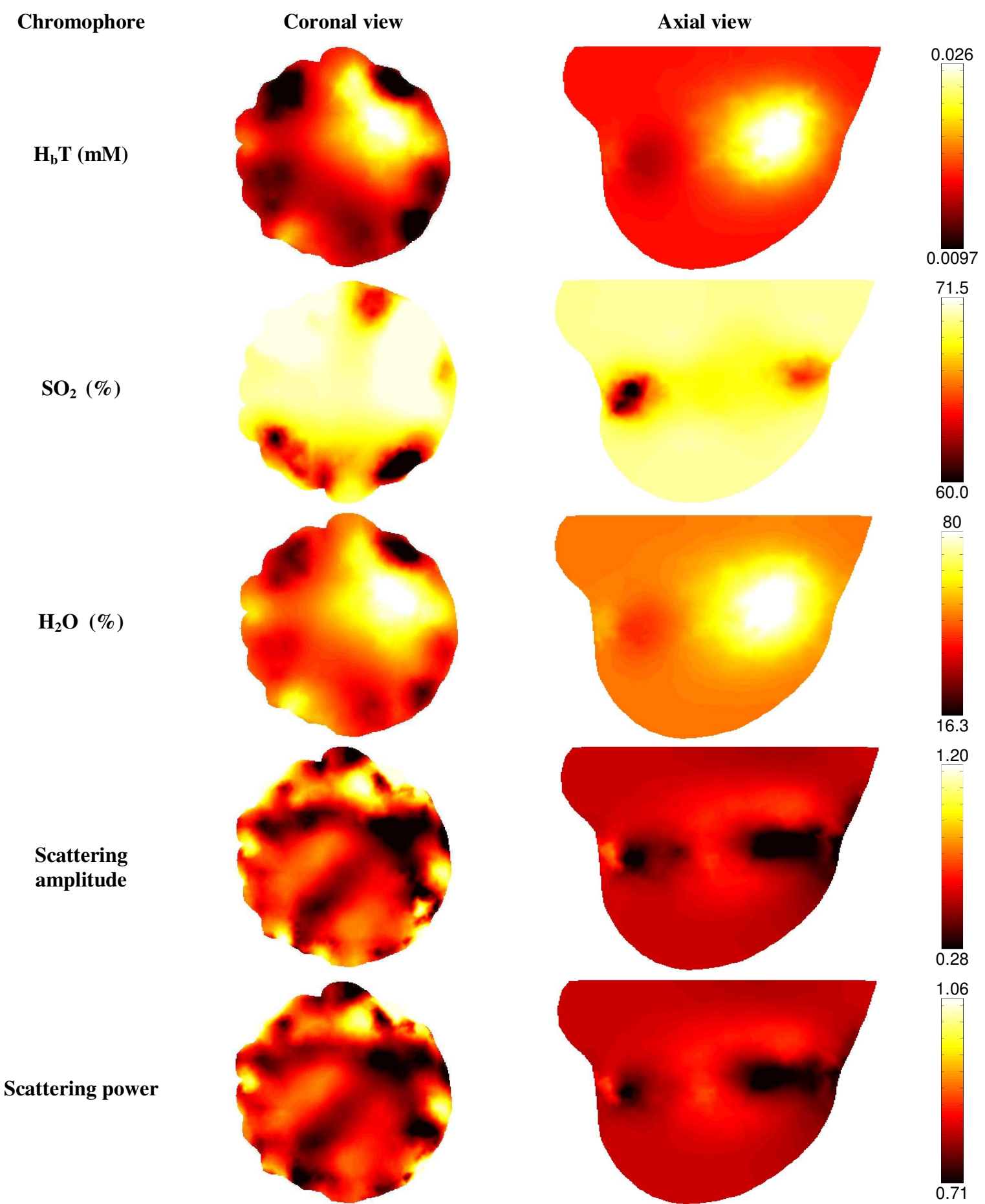

Figure 4. Reconstructed chromophore concentrations of total hemoglobin $\left(\mathrm{H}_{\mathrm{b}} \mathrm{T}\right)$, oxygen saturation $\left(\mathrm{SO}_{2}\right)$, water content $\left(\mathrm{H}_{2} \mathrm{O}\right)$, scattering amplitude and scattering power using the spectral reconstruction displayed as coronal and anatomically axial views. 


\subsection{Spectral case}

Here all six wavelengths are used simultaneously within the reconstruction algorithm. The chromophore concentrations are reconstructed iteratively until the change in the modeled data improved by less than $2 \%$. This process took a computation time of 14150 s and took 3 iterations. The corresponding images are shown in figure 4 . From the images, it is seen that a peak in total hemoglobin of $0.026 \mathrm{mM}$ and oxygen saturation of $63 \%$ is found within the region of interest compared to a background of $0.018 \mathrm{mM}$ and $70 \%$ respectively. A peak in the water content of $80 \%$ is also calculated using this direct spectral method, as compared to the background of 50\%. However, for the reconstructed scattering amplitude and scattering power, noise near the external boundary dominated making any distinction of the nature of the scattering parameters difficult within the region of interest. These results do however agree well to the MRI images shown with regards to the locality of the anomaly and a 2D spatially constrained study which has been previously reported [11].

\section{Conclusion}

The numerical modeling techniques and image reconstructions using NIRFAST are highlighted. It is shown that functional images can be derived from both single wavelength image reconstructions as well as a direct 'spectral' imaging technique. However a direct 'spectral' technique allows a much more computationally efficient method for deriving functional parameters. Additionally, given that data are coupled in a direct spectral method, images are less prone to noise in data and image artifacts. In this work we have carried out reconstructions using NIRFAST for a case study of a 29 year old patient with a $2 \mathrm{~cm}$ infiltrating ductal carcinoma. Images using both single wavelengths and spectral reconstructions are shown. In each case the region of interest was successfully found which corresponded well to the MRI images of the tumor.

\section{References}

1. Gibson, A., Hebden, JC, and Arridge, SR, Recent advances in diffuse optical imaging. Phys. Med. Biol., 2005. 50: p. R1-R43.

2. $\quad$ Arridge, S.R., Optical tomography in medical imaging. Inverse Problems, 1999. 15(2): p. R41-R93.

3. Srinivasan, S., Pogue, B. W., Brooksby, B., Jiang, S., Dehghani, H., Kogel, C., Poplack, S. P., and Paulsen, K. D., Near-Infrared Characterization of Breast Tumors In Vivo using Spectrally-Constrained Reconstruction. Technology in Cancer Research \& Treatment, 2005. 5(5): p. 513-526.

4. Patterson, M.S., B.C. Wilson, and D.R. Wyman, The propagation of optical radiation in tissue I. models of radiation transport and their application. Lasers Med. Sci., 1990. 6: p. 155-168.

5. Hebden, J., et al., Imaging changes in blood volume and oxygenation in the newborn infant brain using threedimensional optical tomography. Physics in Medicine and Biology, 2004. 49: p. 1117-1130.

6. Boas, D.A., et al., Improving the diffuse optical imaging spatial resolution of the cerebral hemodynamic response to brain activation in humans. Opt. Lett., 2004. 29(13): p. 1506-1508.

7. Srinivasan, S., Pogue, B. W., Jiang, S., Dehghani, H., Kogel, C., Soho, S., Gibson, J. J., Tosteson, T. D., Poplack, S. P., Paulsen, K. D., Interpreting Hemoglobin and Water Concentration, Oxygen Saturation and Scattering Measured In Vivo by Near-Infrared Breast Tomography. PNAS, 2003. 100(21): p. 12349-12354.

8. Dehghani, H., Pogue, B. W., Poplack, S. P., Paulsen, K. D., Multiwavelength Three-Dimensional NearInfrared Tomography of the Breast: Initial Simulation, Phantom, and Clinical Results. Applied Optics, 2003. 42(1): p. 135-145.

9. McBride, T.O., et al., Strategies for absolute calibration of near infrared tomographic tissue imaging. Oxygen Transport to Tissue XXIV, 2003: p. 85-99.

10. Brooksby, B., Jiang, S., Kogel, C., Doyley, M., Dehghani, H., Weaver, J. B., Poplack, S. P., Pogue, B. W., Paulsen, K. D., Magnetic Resonance-Guided Near-Infrared Tomography of the Breast. Rev. Sci. Instrum., 2004. 75(12). 
11. Carpenter, C.M., Pogue, B. W., Jiang, S., Dehghani, H., Wang, X., Paulsen, K. D., Wells, W. A., Forero, J., Kogel, C., Weaver, J. B., Poplack, S. P. and Kaufman, P. A., Image-Guided Optical Spectroscopy provides Molecular-Specific Information In Vivo: MRI-Guided Spectroscopy of Breast Cancer Hemoglobin, Water and Scatterer Size. Optics Letters, 2007. 32(8): p. 933-935. 